\title{
Mögliche Massnahmen zur Restaurierung des Sempachersees
}

\author{
Von R. Gächter ${ }^{1}$ ), D. Imboden ${ }^{1}$ ), H. Bührer ${ }^{1}$ ) und P. Stadelmann ${ }^{2}$ ) \\ 1) Eidg. Anstalt für Wasserversorgung, Abwasserreinigung und Gewässerschutz, CH-8600 Dübendorf \\ 2) Kantonales Gewässerschutzamt, $\mathrm{CH}-6003$ Luzern
}

Manuskript eingegangen am 8. April 1983

\begin{abstract}
Since 1954 average orthophosphate and total phosphorus concentrations have increased twenty and eightfold respectively in Lake Sempach. It is demonstrated that the lake is not in steady state with its phosphorus loading and that the net deposition rate of phosphorus is not linearly related to the phosphorus content of the lake. This implies that linear steady state one-box models are unsuitable to describe the phosphorus balance of this lake. Applying a nonlinear dynamic lake model we predict that the defined water quality goals $\left([\overline{\mathrm{P}}] \leqslant 30 \mathrm{mg} \mathrm{m}^{-3},\left[\mathrm{O}_{2}\right] \geqslant 4 \mathrm{mg} \mathrm{m}^{-3}\right)$ can only be achieved within the next 15 years if the external phosphorus loading is reduced by at least $50 \%$ and simultaneously lake-internal measures, such as hypolimnion areation or hypolimnion siphoning are carried into effect.
\end{abstract}

\section{Einleitung}

Der Sempachersee liegt in der Zentralsehweiz, $15 \mathrm{~km} \mathrm{NW}$ von Luzern. Seine Oberfläche beträgt $14,4 \mathrm{~km}^{2}$, die grösste Tiefe $87 \mathrm{~m}$, die mittlere Tiefe $46 \mathrm{~m}$. Das Einzugsgebiet (ohne Seeoberfläche) umfasst $62,6 \mathrm{~km}^{2}$ und ist damit nur rund viermal grösser als die Seeoberfläche. Der Abfluss der Suhre beträgt im Mittel etwa $1,2 \mathrm{~m}^{3} / \mathrm{s}$ (Casiraghi [3]). Um den See über seine natürlichen Zuflüsse neu aufzufüllen, würden etwa 17 Jahre benötigt. Diese sehr lange theoretische Erneuerungszeit des Wassers führt dazu, dass der See nur langsam auf Konzentrationsveränderungen in seinen Zuflüssen reagiert. Im Extremfall (d.h. für einen Stoff mit sehr kleiner Nettosedimentation) dürfte diese Anpassungszeit in der Grössenordnung von etwa 50 Jahren liegen. Die langsame Reaktion des Sees auf Veränderungen in der Zuflussqualität ist für den See von Vorteil, wenn sich die Zuflussqualität verschlechtert, wirkt sich aber zum Nachteil aus, wenn der See durch eine Verbesserung der Zuflussqualität saniert werden sollte.

Im Jahre 1894 untersuchte Heuscher [7] als erster den Sempachersee. Bemerkenswert ist, dass er von einer grossen Armut an Wasserpflanzen spricht und keinen einzigen Vertreter der Laichkräuter (Potamogetonacea) erwähnt. Niedrige Besiedlungsdichten und das Fehlen von Potamogetonen sind Zeichen dafür, dass der See 1894 noch ein oligotrophes Gewässer war. Dies muss im wesentlichen auch noch in den Jahren 1932 bis 1934 zugetroffen haben, wo in Tiefen von 6 bis $8 \mathrm{~m}$ Charawiesen beschrieben wurden (Du Bois und Geigy [4]), denn Characeen werden in diesen 
Tiefen nur in nährstoffarmen Gewässern angetroffen. Allerdings beschreiben diese Autoren bereits fakultative Vorkommen von Potamogetonen in der Uferzone und kommen auch aufgrund ihrer Untersuchung der Bodenfauna zum Schluss, der See befinde sich in einem Übergangsstadium von Oligotrophie zu Eutrophie. Mit Hilfe von Sedimentuntersuchungen konnte Züllig [14] ein seit 1947 zunehmendes Auftreten von «Faulschlamm» nachweisen, ein Indiz dafür, dass seit diesem Zeitpunkt das auf den Seegrund abgesunkene Plankton nicht mehr vollständig aerob mineralisiert worden ist.

Eine Zusammenstellung der limnologischen Literatur über den Sempachersee von 1869 bis 1980 kann einer Veröffentlichung von Stadelmann [11] entnommen werden.

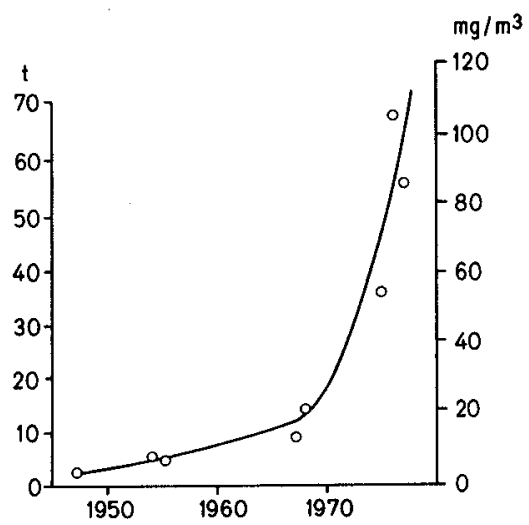

Abb. 1. Veränderung des Phosphatinhalts und der mittleren Phosphatkonzentration $\left(\mathrm{mg} / \mathrm{m}^{3}\right) \mathrm{im}$ Sempachersee seit 1947.

Figure 1. Changes of phosphate content (t) and average phosphate concentrations $\left(\mathrm{mg} / \mathrm{m}^{-3}\right)$ in Lake Sempach since 1947.

Abb. 1 zeigt die Veränderung des Phosphatgehalts im See seit 1947. Die jeweils im November erhobenen Messungen lassen mit erschreckender Deutlichkeit erkennen, dass die bereits getroffenen Gewässerschutzmassnahmen, nämlich der Bau der Kläranlagen Eich (Baujahr 1968), Gunzwil (1970), Sempach (1971) und Hildisrieden (1973), welche 1976/77 das Abwasser von 5681 Einwohnern (58\% der Gesamtbevölkerung) reinigten, bei weitem nicht ausreichten, die sich seit 1950 abzeichnende Eutrophierung auch nur zu verlangsamen, geschweige denn zu stoppen.

Leider liegen für die gleiche Zeitperiode keine Daten über die Primärproduktion des Sees vor. Die zeitliche Veränderung verschiedener in Abb. 2 zusammengestellter Parameter zeigt jedoch klar, dass seit 1940 sowohl die Primärproduktion als auch die hypolimnische Destruktion von organischem Material deutlich zugenommen haben.

Ziel dieser Arbeit ist es, die zur Sanierung des Sees notwendige Basisinformation zusammenzustellen, d.h.

- seine aktuelle Phosphorbelastung zu quantifizieren und einzelne Phosphorquellen soweit als möglich zu identifizieren, 

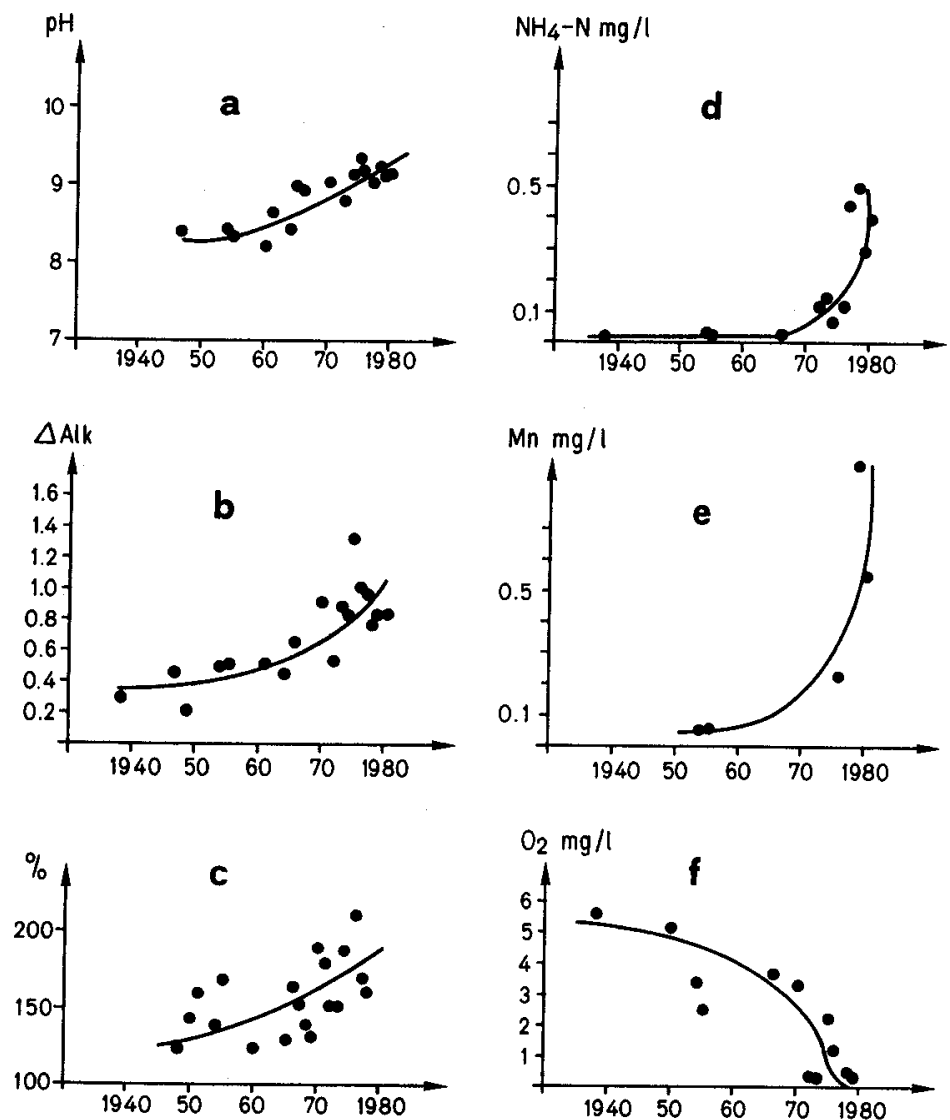

Abb. 2. a: Im Epilimnion beobachtete pH-Maxima. b: Epilimnische Alkalinitätsdifferenz zwischen Vollzirkulation und Spätsommer. c: Epilimnische Sauerstoffübersättigung. d: Ammoniumkonzentration in $80 \mathrm{~m}$ Tiefe im Oktober. e: Mangankonzentration in $80 \mathrm{~m}$ Tiefe im Oktober. f: Sauerstoffkonzentration in $80 \mathrm{~m}$ Tiefe im Oktober.

Figure 2. $\mathrm{pH}$ maxima (a), differences in alkalinity between winter and summer (b) and supersaturation of oxygen (c) in the epilimnion. Concentrations of ammonia (d), manganese (e) and oxygen (f) observed in October at a depth of $80 \mathrm{~m}$.

- den Zusammenhang zwischen externer Phosphorbelastung und Phosphorkonzentration im See aufzuzeigen,

- mit Hilfe eines dynamischen Seenmodells (Imboden et al. [9]) abzuschätzen, wie der See auf eine veränderte Phosphorbelastung und (oder) seeinterne Massnahmen, wie Belüftung oder Hypolimniondränage, reagieren würde.

Prognosen über die Auswirkung verschiedener Massnahmen auf einen See sind wertvolle Hilfsmittel zur Evaluation einer optimalen Gewässerschutzstrategie. Sie verhindern, dass Fehlinvestitionen getätigt oder in getroffene Massnahmen falsche Erfolgserwartungen gesetzt werden. 


\section{Phosphorbilanz des Sempachersees}

\subsection{Quantifizierung der Phosphorbelastung}

Die Phosphorbelastung des Sempachersees, welche in den letzten 25 Jahren dreimal abgeschätzt wurde, hat sich in diesem Zeitabschnitt nahezu verfünffacht (Tab. 1 ).

Tabelle 1. Gesamt-P-Belastung des Sempachersees in den Jahren 1954, 1966/67, 1976/77.

Table 1. Total P-loading of lake Sempach in 1954, 1966/67 and 1976/77.

\begin{tabular}{lll}
\hline Jahr & P-Belastung & Referenz \\
\hline 1954 & $3,4 \mathrm{t} / \mathrm{Jahr}^{-1}$ & Thomas, unveröffentlicht \\
$1966 / 67$ & $9,2 \mathrm{t} / \mathrm{Jahr}^{-1}$ & Kantonales Amt für Gewässerschutz, \\
& unveröffentlicht \\
$1976 / 77$ & Gutachten der EAWAG über die \\
& Sanierungsmöglichkeiten des Sempachersees (1979) \\
& {$[5]$} \\
\hline
\end{tabular}

I) davon 1,2 t/Jahr durch direkten Niederschlag auf die Seeoberfläche.

In Tabelle 2 wurde versucht, für diesen Zeitraum die zeitliche Veränderung der abwasserbürtigen Phosphorfraktion abzuschätzen. Dank den bisher getroffenen Gewässerschutzmassnahmen ist die abwasserbürtige P-Belastung des Sees seit 1970 nicht mehr angestiegen, sondern sogar niedriger geworden. Nach Tabelle 2 beseitigten 1977 noch 20\% der Bevölkerung ihr Abwasser über Klärgruben mit Überlauf, praktisch ohne Rückhalteeffekt bezüglich Phosphor. Über die Abwasserbeseitigungstechnik von weiteren $22 \%$ der Einwohner bestand Unklarheit.

Der Beitrag der Landwirtschaft zur Gesamtphosphorbelastung des Sees lässt sich durch Differenzbildung zwischen der gemessenen Gesamt-P-Belastung (Tab. 1) und den geschätzten Abwasserphosphorfrachten (Tab.2) abschätzen. Danach haben Bodenerosion und Eluation im Jahre 1954 0,1 t/Jahr, 1967 höchstens 2,1 t/Jahr-1 und 1976/77 mindestens 4,2 $\mathrm{t} / \mathrm{Jahr}^{-1}$ und höchstens $8,0 \mathrm{t} / \mathrm{Jahr}^{-1}$ zur P-Belastung des Sees beigetragen. Auch wenn man berücksichtigt, dass sowohl die direkten Bestimmungen des Phosphoreintrags (Tab.1) als auch die Schätzungen über die abwasserbürtige Fracht (Tab.2) mit Fehlern behaftet sind, so zeigt diese Differenzrechnung doch klar, dass die bodenbürtige Phosphorbelastung des Sees seit 1954 deutlich zugenommen hat. Im Einzugsgebiet des Sees sind rund $10 \mathrm{~km}^{2}$ bewaldet, und $52 \mathrm{~km}^{2}$ werden landwirtschaftlich genutzt. Da die Phosphorbelastung des Sees durch den Wald ( $5 \mathrm{~kg} \mathrm{~km}^{-2} \mathrm{Jahr}$ ) vernachlässigbar klein ist, muss der durch Eluation und Erosion bewirkte Phosphorverlust der landwirtschaftlich genutzten Gebiete heute im Mittel 80 bis $150 \mathrm{~kg} \mathrm{~km}^{-2} \mathrm{Jahr}^{-1}$ betragen. Diese Werte liegen deutlich über den von Gächter und Furrer [6] fürs schweizerische Mittelland ermittelten Werten von $35 \mathrm{~kg} / \mathrm{km}^{2} \mathrm{Jahr}$.

Es wird vermutet, dass diese extrem hohen Exportkoeffizienten im Zusammenhang mit dem in den letzten Jahren stark angestiegenen Schweinebestand im Einzugsgebiet des Sees stehen. 1975 wurden rund 40000 Schweine und 13000 Rinder gezählt (Boog, mündliche Mitteilung). Eine Phosphorbilanz im Einzugsgebiet des Sees hat ergeben, dass jährlich rund 250 Tonnen $P$ in Form von Hofdünger und 60 Tonnen $P$ als Mineraldünger ausgebracht werden. Vergleicht man diese ausgebrachte Dünger- 
Tabelle 2. Bevölkerungsentwicklung und Anschluss der Einwohner an Kläranlagen im Einzugsgebiet des Sempachersees. Frachtangaben in t/Jahr. Table 2. Development of the population and its connection to wastewater treatment plants in the catchment area of Lake Sempach. Load in $t /$ year.

\begin{tabular}{|c|c|c|c|c|c|c|c|c|c|c|c|c|c|c|}
\hline \multirow[t]{3}{*}{ Jahr } & \multirow{3}{*}{$\begin{array}{l}\text { Einwohne } \\
\text { total }\end{array}$} & \multirow{3}{*}{$\begin{array}{l}\text { Fracht } \\
\text { koeffizient } \\
(6)\end{array}$} & \multicolumn{3}{|c|}{ An ARA angeschlossen } & \multicolumn{3}{|c|}{ Klärgruben mit Überlauf ${ }^{1}$ ) } & \multirow{2}{*}{\multicolumn{4}{|c|}{$\begin{array}{l}\text { Abwasserbeseitigung } \\
\text { Jauchegrube ohne Überlauf oder } \\
\text { mit nichtbewilligtem Überlauf } \\
\text { Geschätzte P-Fracht }\end{array}$}} & \multirow{2}{*}{\multicolumn{2}{|c|}{$\begin{array}{l}\text { Geschätzte Abwasser } \\
\text { P-Fracht total }\end{array}$}} \\
\hline & & & \multirow[t]{2}{*}{ Absolut } & \multirow[t]{2}{*}{$\%$} & \multirow[t]{2}{*}{ P.Fracht } & \multirow[t]{2}{*}{ Absolut } & \multirow[t]{2}{*}{$\%$} & \multirow[t]{2}{*}{ P-Fracht } & & & & & & \\
\hline & & & & & & & & & Absolut & $\%$ & Minimal' $^{2}$ ) & $\left.\operatorname{Maximal}^{1}\right)$ & Minimal & Maximal \\
\hline 1950 & 6475 & 920 & - & - & - & 3530 & 55 & 3,3 & 2945 & 45 & 0 & 2,7 & 3,3 & 6,0 \\
\hline 1967 & 6910 & 1620 & - & - & - & 4405 & 64 & 7,1 & 2505 & 36 & 0 & 4,1 & 7,1 & 11,2 \\
\hline 1970 & 7211 & 1790 & 300 & 5 & $\left.0,4^{3}\right)$ & 4580 & 63 & 8,2 & 2331 & 32 & 0 & 4,2 & 8,6 & 12,8 \\
\hline 1975 & 9514 & 1790 & 4375 & 46 & $\left.1,7^{4}\right)$ & 2660 & 28 & 4,8 & 2479 & 26 & 0 & 4,4 & 6,5 & 10,9 \\
\hline $1976 / 77$ & 9790 & 1790 & 5681 & 58 & $\left.2,0^{5}\right)$ & 1960 & 20 & 3,5 & 2149 & 22 & 0 & 3,8 & 5,5 & 9,3 \\
\hline
\end{tabular}

1) Kein wirksamer Rückhalt von $P$

2) Annahme: kein Überlauf.

3) Annahme: Eliminationseffekt Kläranlage Eich 30\%.

4) Annahme: $30 \%$ Eliminationseffekt in Kläranlage Eich und Gunzwil (525 EW) und 85\% Eliminationseffekt in Kläranlage Sempach,

Hildisrieden ( $3950 \mathrm{EW}$ )

5) Annahme: $30 \%$ Eliminationseffekt in Kläranlage Eich und Gunzwil (660 EW), 85\% Eliminationseffekt in Kläranlage Hildisrieden, Sempach

(4291 EW), und 100\% Elimination ARA Suhrental (730 EW).

6) Sly [8], Angaben in $\mathrm{g} \mathrm{PKopf}^{-1} \mathrm{Jahr}^{-1}$. 
menge von mindestens $310 \mathrm{t} / \mathrm{Jahr}$ mit den davon in die Gewässer gelangenden Phosphormengen von 4,1 bis $7,9 \mathrm{t} / \mathrm{Jahr}$, so zeigt es sich, dass weniger als $3 \%$ des ausgebrachten Düngers in den See gelangen. Derartige Düngerverlustraten sind aus der Sicht der Landwirtschaft begreiflicherweise vernachlässigbar klein. Für den Sempachersee, der zusätzlich auch noch mit Abwasser belastet wird, ist diese «Grundlast» aber bereits gefährlich hoch. Es müssen daher wegen der Ansprüche des Gewässerschutzes Mittel und Wege gefunden werden, die durch die Landwirtschaft und Bioindustrie verursachte P-Belastung wesentlich zu verringern.

Bemerkenswert ist, dass sich bei der heutigen landwirtschaftlichen Praxis der Phosphorgehalt des Bodens jährlich um durchschnittlich $2400 \mathrm{~kg} / \mathrm{km}^{2}$ erhöht (AGBA) [1]. Es besteht daher die Gefahr, dass selbst bei gleichbleibender Düngepraxis die aus Eluation und Erosion herrührende Phosphorbelastung des Sees noch weiter ansteigen wird.

Langfrisig muss man sich das Ziel setzen, den bodenbürtigen P-Export auf $50 \mathrm{~kg}$ / $\mathrm{km}^{2} \mathrm{Jahr}$ zu verringern, d. h. auf einen Wert, der noch deutlich über den Erfahrungswerten für das schweizerische Mittelland liegt $\left(35 \mathrm{~kg} / \mathrm{km}^{2} \mathrm{Jahr}\right)$ und daher den steileren Lagen der landwirtschaftlichen Betriebe im Einzugsgebiet des Sempachersees Rechnung trägt.

Tabelle 3. P-Belastung des Sempachersees nach vollständiger Sanierung des Einzugsgebiet.

Table 3. P loading of Lake Sempach after complete restoration of the catchment area.

\begin{tabular}{ll}
\hline Niederschläge & $1,2 \mathrm{t} / \mathrm{Jahr}$ \\
Abwässer (nach vollständiger Sanierung) & $3 \mathrm{t} / \mathrm{Jahr}$ \\
Export aus landwirtschaftlich genutzten Gebieten $\left(52 \mathrm{~km}^{2} \times 50 \mathrm{~kg} /\right.$ & $2,6 \mathrm{t} / \mathrm{Jahr}$ \\
\hline $\left.\mathrm{km}^{2} \mathrm{Jahr}\right)$ & $6,8 \mathrm{t} / \mathrm{Jahr}$ \\
\hline Totalfracht & \\
\hline
\end{tabular}

Mit einer vollständigen Sanierung der häuslichen Abwässer (Anschluss an eine ARA mit 85\% P-Rückhalt oder Sammlung in abflusslosen Gruben und sorgfältige landwirtschaftliche Verwertung) liesse sich die heutige jährliche Abwasserphosphorfracht von 5,5 bis 9,3 t auf rund $3 \mathrm{t}$ verringern. Wie Tabelle 3 zeigt, wäre es somit unrealistisch anzunehmen, die jährliche Gesamtphosphorbelastung des Sees könne in naher Zukunft auf weniger als $7 \mathrm{t}$ vermindert werden.

\subsection{Zusammenhang zwischen P-Konzentration und P-Verlust via Abfluss und Sedimentation}

Der Zusammenhang zwischen Phosphorzufuhr (Z), Phosphorinhalt des Sees (I), Export via den Abfluss (E) und Nettosedimentation (S) kann dargestellt werden durch die Gleichung

$$
\frac{\mathrm{dI}}{\mathrm{dt}}=\mathrm{Z}-\mathrm{E}-\mathrm{S} \text {. }
$$

Unter der Nettosedimentation $\mathrm{S}$ verstehen wir die Differenz zwischen der PEinlagerung ins Sediment via Partikelsedimentation oder Adsorption an der Sedimentoberfläche und der P-Rücklösung von Sediment. 
Der P-Export berechnet sich aus der P-Konzentration an der Seeoberfläche multipliziert mit der Wassermenge im Abfluss. Da aber offenbar zwischen der oberflächlichen und mittleren P-Konzentration (bzw. der P-Menge im See) ein linearer Zusammenhang besteht, kann für den Export $\mathrm{E}$ die lineare Beziehung

$$
\mathrm{E}=a \cdot \mathrm{I}
$$

benützt werden. Nach Abb. 3 ergibt sich für $a$ der Wert 0,042 $\mathrm{Jahr}^{-1}$, obwohl der jährliche Wasseraustausch $0,057 \mathrm{Jahr}^{-1}$ beträgt. Der Grund für die gegenüber dem Wasser verkleinerte spezifische Exportrate liegt darin, dass während der Sommerstagnation die Seeoberfläche an Phosphor verarmt, der Phosphor sich also in den tieferen Schichten ansammelt und dort vom Export durch den Abfluss abgeschirmt bleibt.

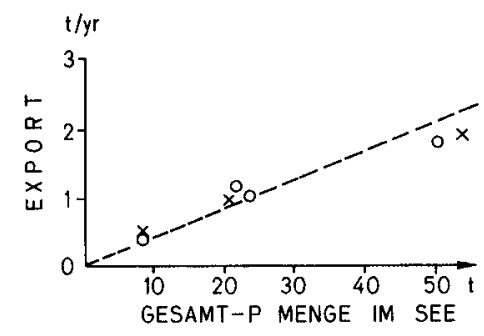

Abb. 3. Zusammenhang zwischen dem Phosphorexport via Abfluss und dem Phosphorinhalt des Sees. o: Export $=\bar{Q} \cdot[\bar{P}]$, wobei $\bar{Q}$ den mittleren Jahresabfluss $\left(3,7 \cdot 10^{7} \mathrm{~m}^{3} / \mathrm{Jahr}^{-1}\right)$ und $[\bar{P}]$ die mittlere Phosphorkonzentration an der Oberfläche bedeutet. $X:$ Export $=Q \cdot[\bar{P}]$, wobei $Q$ die aktuell gemessene Abflussrate bezeichnet.

Figure 3. Relationship between phosphorus export via effluent and phosphorus content of the lake.

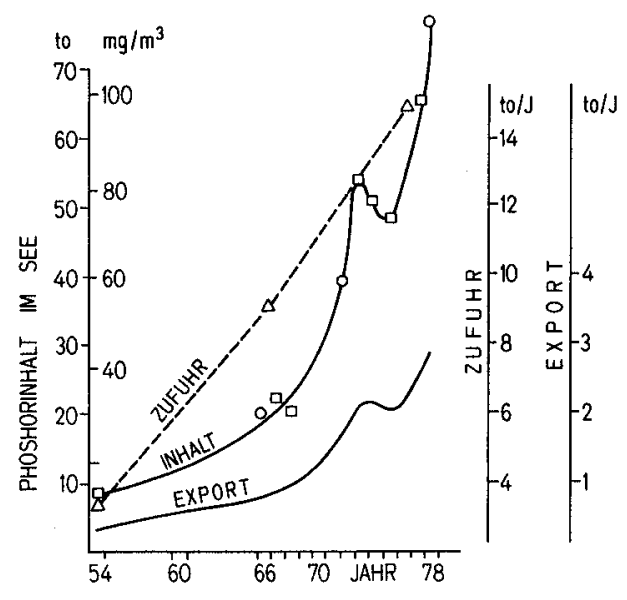

Abb.4. Zeitliche Veränderung des Gesamtphosphorinhalts, des Exports (-) und der Phosphorbelastung des Sempachersees (---).

Figure 4. Changes of input (--), content (INHALT) and export (-) of total phosphorus in Lake Sempach. 


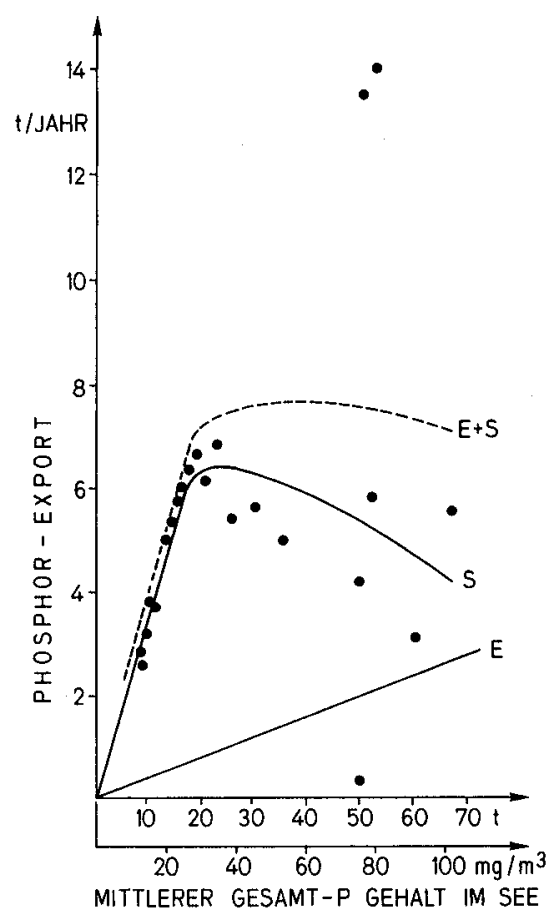

Abb. 5. Zusammenhang zwischen Phosphorinhalt, -Export (E) und -Nettosedimentation (S) im Sempachersee.

Figure 5. Relationship between phosphorus content, phosphorus export (E) and net deposition of phosphorus (S) in Lake Sempach.

Die mittleren jährlichen Nettosedimentationsraten $\mathrm{S}$ wurden für den Zeitabschnitt 1954 bis 1976 mit Hilfe der Gleichung

$$
\mathrm{S}=\mathrm{Z}-\alpha \cdot \mathrm{I}-\frac{\mathrm{dI}}{\mathrm{dt}}
$$

berechnet. Dabei bedeuten $\mathrm{Z}$ und I die mittlere jährliche Zufuhr und den mittleren Phosphorinhalt des Sees während des entsprechenden Jahres und dI/dt den PAnstieg im See während des Jahres. Diese Grössen können aus den Abb. 3 und 4 entnommen werden.

In Abb. 5 wurden die Nettosedimentationsraten gegen die im See vorhandene Phosphormenge aufgetragen. Es zeigt sich, dass Seemodelle, die zwischen der Nettosedimentationsrate und dem Seeinhalt einen linearen Zusammenhang postulieren (Vollenweider [13]), dem Phosphorhaushalt des Sempachersees nur bis zu Phosphorkonzentrationen von höchstens $30 \mathrm{mg} \mathrm{m}^{-3}$ gerecht werden. Oberhalb dieser kritischen Konzentration treten zwischen den Grössen «Nettosedimentation» und «mittlere Seekonzentration» starke Streuungen auf. Diese mögen zum Teil damit erklärt werden, dass die Geschwindigkeit der Phosphorfreisetzung am 


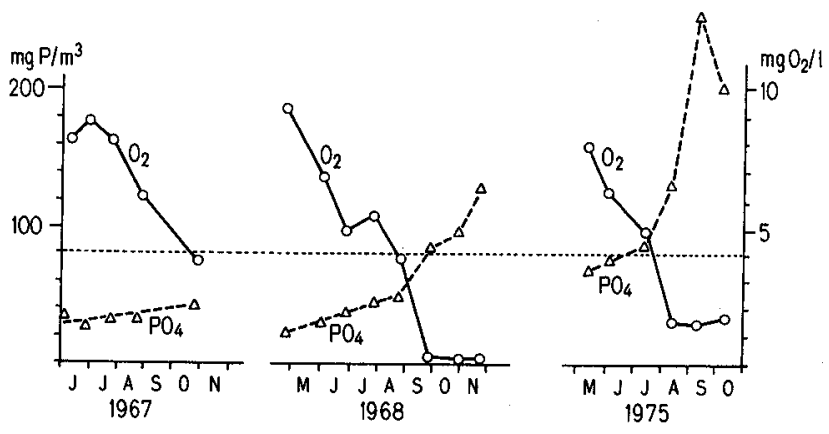

Abb.6. Sauerstoffzehrung und Phosphatakkumulierung in $85 \mathrm{~m}$ Tiefe während der Jahre 1965, 1968 und 1975 in $85 \mathrm{~m}$ Tiefe.

Figure 6. Depletion of oxygen and accumulation of phosphate at a depth of $85 \mathrm{~m}$.

Seegrund äusserst empfindlich von der Sauerstoffkonzentration (Abb.6) und damit von den von Jahr zu Jahr variablen physikalisch-meteorologischen Bedingungen abhängt. Trotz den erheblichen Streuungen kommt aber klar zum Ausdruck, dass oberhalb des Grenzwerts von etwa $30 \mathrm{mg} \mathrm{P} / \mathrm{m}^{3}$ die Nettosedimentation im Mittel nicht mehr weiter ansteigt, sondern mit zunehmender Konzentration eher wieder kleiner wird. Diese Veränderung in der Charakteristik der Nettosedimentation ist neben der stets steigenden externen P-Zufuhr wesentlich für den rasanten P-Anstieg seit 1968 mitverantwortlich.

\section{3. Überprüfung der Erfolgsaussichten von möglichen Gewässerschutzmassnahmen mit Hilfe eines Seemodells}

Die für Seen erwünschte Wasserqualität wird in der Verordnung über Abwassereinleitungen, einer Verordnung zum schweizerischen Gewässerschutzgesetz [12], wie folgt umschrieben:

- «Der biologische Zustand der Freiwasserzone soll höchstens dem mesotrophen Produktionstypus entsprechen.» Eine Interpretation dieser Zielsetzung ergibt, dass die epilimnische Gesamtphosphorkonzentration $30 \mathrm{mg} \mathrm{m}^{-3}$ nie überschreiten und die Primärproduktionsrate niedriger als $150 \mathrm{~g} \mathrm{C} \mathrm{m}^{-2} \mathrm{Jahr}^{-1}$ sein sollte.

- «Der Sauerstoffgehalt soll zu keiner Zeit und in keiner Tiefe weniger als $4 \mathrm{mg}$ $\mathrm{O}_{2} / 1$ betragen.»

Um zu überprüfen, mit welchen Massnahmen diese Ziele erreicht werden könnten, wurde das an der EAWAG entwickelte und in der Literatur beschriebene eindimensionale vertikale Seemodell (Imboden et al. [9]) benützt.

\subsection{Das Modell}

Das Modell (s. Abb. 7) beschreibt die Phosphor- und die Sauerstoffkonzentration in Abhängigkeit von Seetiefe und Jahreszeit, indem die Prozesse «Photosynthese» (Bildung von Biomasse, bei gleichzeitiger Sauerstoffproduktion), «Mineralisation» (Destruktion von Biomasse unter Sauerstoffverbrauch), «Sedimentation» und «Sau- 


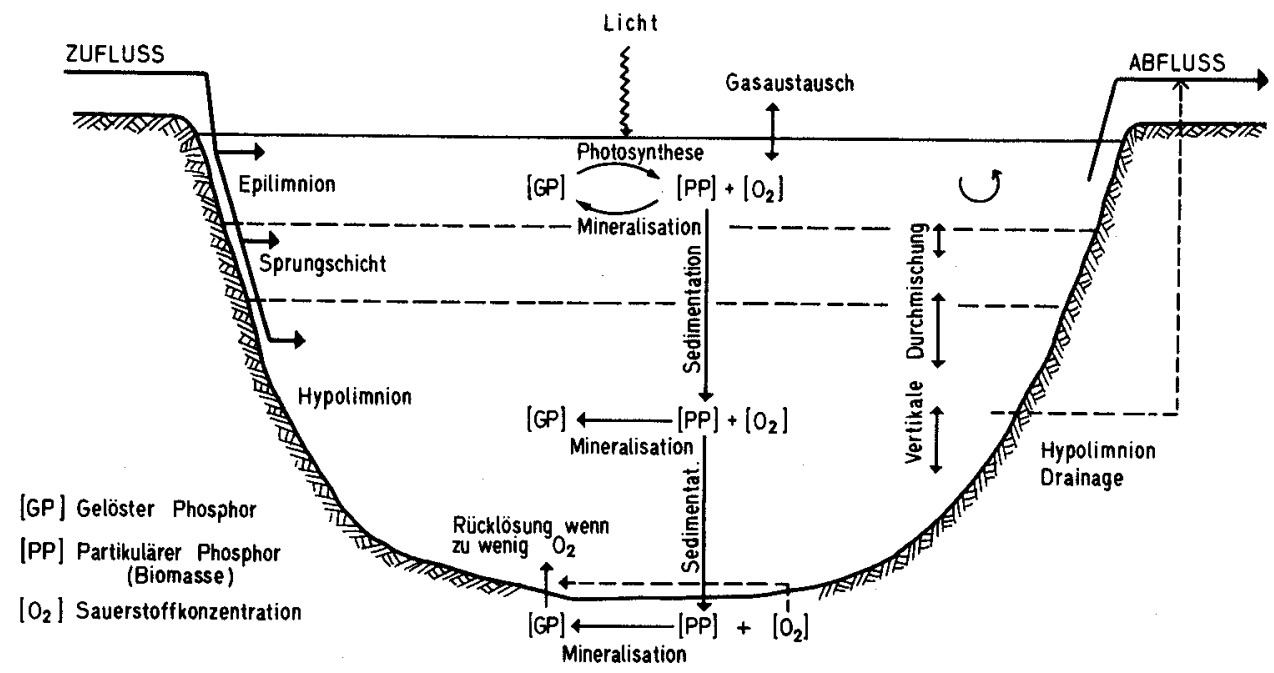

Abb. 7. Modell zur Beschreibung von Photosynthese, Biomasse, Phosphat- und Sauerstoffkonzentration im See und im oberen Sediment, modifiziert nach Imboden und Gächter [10].

Figure 7. Lake model to describe photosynthesis, biomass and concentration of phosphate and oxygen in the lake and at the sediment-water interface. Modified from Imboden and Gächter [10].

erstoffaustausch an der Wasser-Luft-Grenzschicht» mathematisch simuliert werden. Das Modell basiert auf folgenden physikalischen, chemischen und biologischen Eingaben:

1. Seegeometrie: Fläche des Sees an der Oberläche und in zunehmender Tiefe.

2. Tiefe des Epilimnions in Abhängigkeit der Jahreszeit.

3. Dicke der Sprungschicht.

4. Wassertemperatur an der Seeoberfläche in Abhängigkeit der Jahreszeit.

5. Intensität des vertikalen Austauschs in der Sprungschicht und im Hypolimnion (Eddy-Diffusionskoeffizient).

6. Wasserführung der Zuflüsse in Abhängigkeit von der Jahreszeit.

7. Einschichtungstiefe der Zuflüsse.

8. Austauschkoeffizient des Sauerstoffs an der Luft-Wasser-Grenzschicht.

9. Höhe der Seeoberfläche über Meer.

10. Phosphatkonzentration der Zuflüsse, evtl. in Abhängigkeit ihrer Wasserführung.

11. Wachstumsraten in Abhängigkeit von der Phosphatkonzentration und der Jahreszeit (Licht und Wassertemperatur).

12. Extinktion der photosynthetisch aktiven Strahlung durch das Wasser und die suspendierte Biomasse.

13. Mineralisationsraten im Epilimnion, Metalimnion, Hypolimnion und am Seegrund.

14. Sedimentationsgeschwindigkeit der Biomasse im Epilimnion, Metalimnion und Hypolimnion.

15. Kritische Sauerstoffkonzentration, unterhalb der es zur verstärkten Phosphatfreisetzung an der Sediment-Wasser-Kontaktzone kommt. 
16. Relativer Anteil des durch Mineralisation an der Sedimentoberfläche freigesetzten Phosphors, der auch bei höheren Sauerstoffkonzentrationen ins Hypolimnion diffundiert.

Mit Hilfe des oben beschriebenen Seemodells haben wir die zukünftige Entwicklung des Sempachersees unter folgenden Annahmen vorauszusagen versucht:

- Keine seeinternen Massnahmen: Entwicklung bei konstanter bzw. unterschiedlich reduzierter Phosphorbelastung.

- Zwangsmischung (Winter) und Belüftung (Sommer) in Kombination mit verschiedener externer P-Belastung.

- Tiefenwasserableitung in Kombination mit verschiedener externer P-Belastung.

In den Abb.9-11 sind die Resultate in einheitlicher Form dargestellt.

\subsection{Anpassung des Modells an den Ist-Zustand}

Nicht alle für das Modell benötigten Eingabeparameter lassen sich direkt aus vorhandenen Daten ermitteln. Zu den unbestimmten Grössen gehören insbesondere die Sedimentations- und Mineralisationsraten sowie jene Parameter, welche den Phosphoraustausch zwischen Wasser und Sediment beschreiben.

Das Modell wurde geeicht, indem durch sinnvolle Veränderung dieser Parameter versucht wurde, eine möglichst weitgehende Übereinstimmung der räumlichen und zeitlichen Veränderungen der Phosphat- und Sauerstoffkonzentrationen sowie der Primärproduktionsrate zu erzielen.

Als Beispiel sind in Abb. 8 die für den Sommer 1975 berechneten und die im See beobachteten Messwerte für Phosphat und Sauerstoff einander gegenübergestellt. Für Phophat konnte eine sehr gute Übereinstimmung zwischen Modell und Wirklichkeit erzielt werden. Dagegen fielen die berechneten Sauerstoffkonzentrationen etwas zu niedrig aus. Das mag damit zusammenhängen, dass das angenommene feste stöchiometrische Verhältnis von $\Delta \mathrm{O}_{2} / \Delta \mathrm{P}$ von 138 (Redfield [10]) für den Sempachersee nicht exakt zutrifft.

Ein besonderes Problem stellt sich bei der Modellierung des jährlichen Stratifizierungszyklus: Der Sempachersee wird im Winter im Mittel nur ungefähr jedes zweite Jahr vollständig durchmischt. Der Grund dafür liegt bei den von Jahr zu Jahr unterschiedlichen meteorologischen Bedingungen, welche ihrerseits natürlich nicht vorausgesagt werden können. Für unsere Prognosen haben wir eine regelmässige Folge von vollständigen bzw. unvollständigen Mischungszuständen angenommen. Dieser Zyklus ist in den folgenden Abbildungen insbesondere bei den $\mathrm{O}_{2}$-Konzentrationen sichtbar.

\subsection{Prognosen}

Nach Tabelle 1 hat die Phosphorbelastung des Sees in den letzten zwei Jahrzehnten stetig zugenommen und sich mehr als vervierfacht. Es wäre daher bereits als ein Teilerfolg zu werten, wenn es gelänge, einen weiteren Anstieg der Belastung zu verhindern, d.h. die Gesamtphosphorbelastung auf 14,7 t/Jahr zu stabilisieren. 

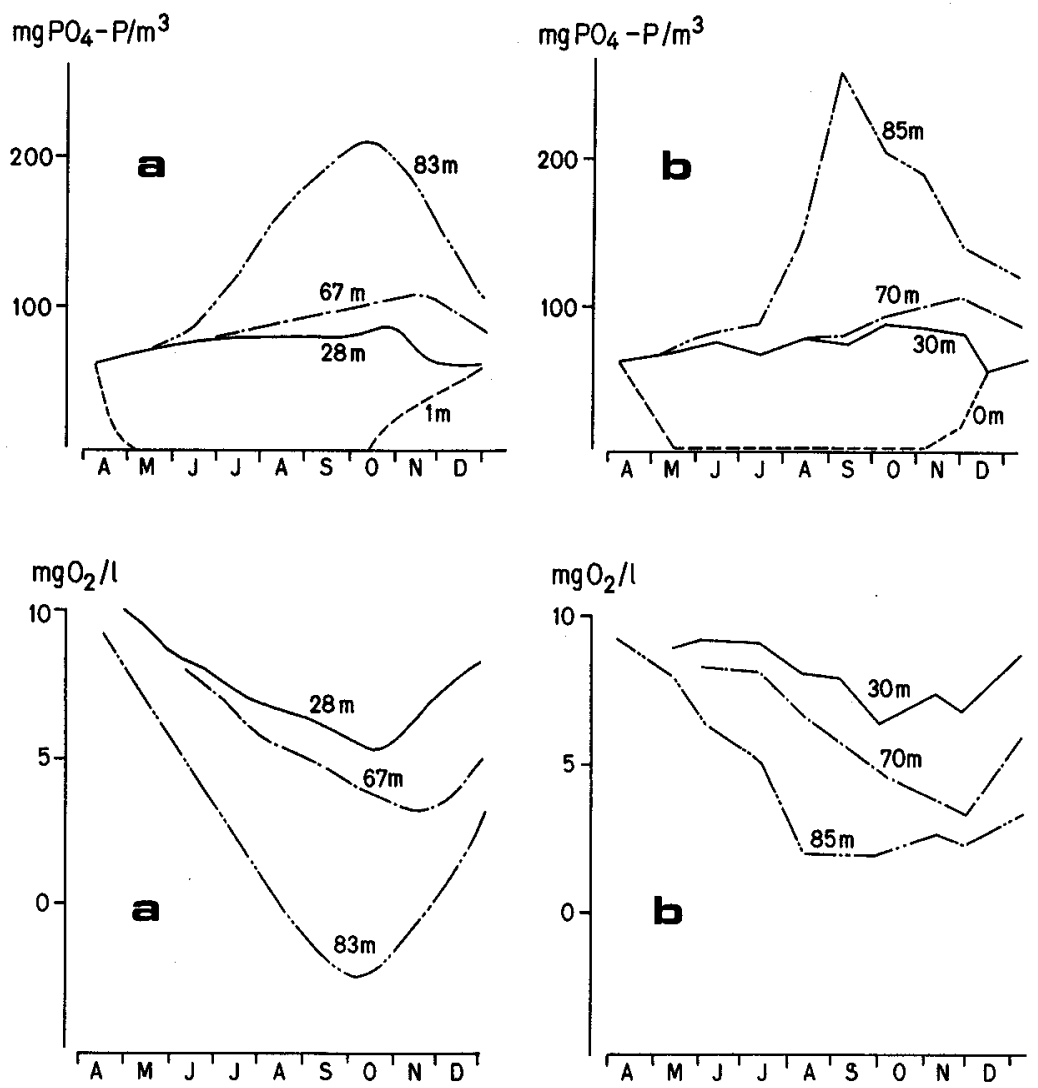

Abb. 8. Vergleich von Modellsimulationen (a) mit effektiv während der Sommerstagnation 1975 gemessenen Phosphat- und Sauerstoffkonzentrationen (b).

Figure 8. Comparison of modeled and measured oxygen and phosphate concentrations.

Nach Abb.9a würde bei gleichbleibender Belastung die Phosphorkonzentration im See weiter ansteigen. Eine Reduktion der Belastung auf die Hälfte hätte wenigstens die Stabilisierung der P-Konzentration auf dem heutigen Niveau zur Folge (Abb.9b). Allerdings würde selbst eine Reduktion der Phosphorbelastung um 75\% nicht ausreichen, das Sanierungsziel (Phosphorkonzentration im See $=20$ bis $30 \mathrm{mg} / \mathrm{m}^{3}$ ) zu erreichen (Abb. 9c), zumindest nicht innerhalb von 10 Jahren.

Mit einer Hypolimniondränage von $1,1 \mathrm{~m}^{3} / \mathrm{s}$ könnte während einer Sommerstagnation von 240 Tagen ein Wasservolumen von $2,3 \cdot 10^{7} \mathrm{~m}^{3}$ abgesaugt werden, wodurch während des Sommers das Wasser zwischen 87 und $75 \mathrm{~m}$ Tiefe einmal ausgetauscht würde. Trotz diesen Massnahmen würde sich die Qualität des Sees bei gleichbleibender P-Belastung weiterhin langsam verschlechtern (Abb. 10). Bei gleichzeitiger Reduktion der Phosphorbelastung um 50\% würden bis zum Jahr 1990/91 die Gesamtphosphorkonzentration im See auf etwa $40 \mathrm{mg} / \mathrm{m}^{3}$ und die Jahresproduktion auf rund $140 \mathrm{mg} \mathrm{C} \mathrm{m}^{-2} \mathrm{Jahr}^{-1}$ absinken (Abb. $10 \mathrm{~b}$ ). Allerdings würden diese Massnahmen nicht ausreichen, um in Jahren mit schwacher Winterdurchmischung 

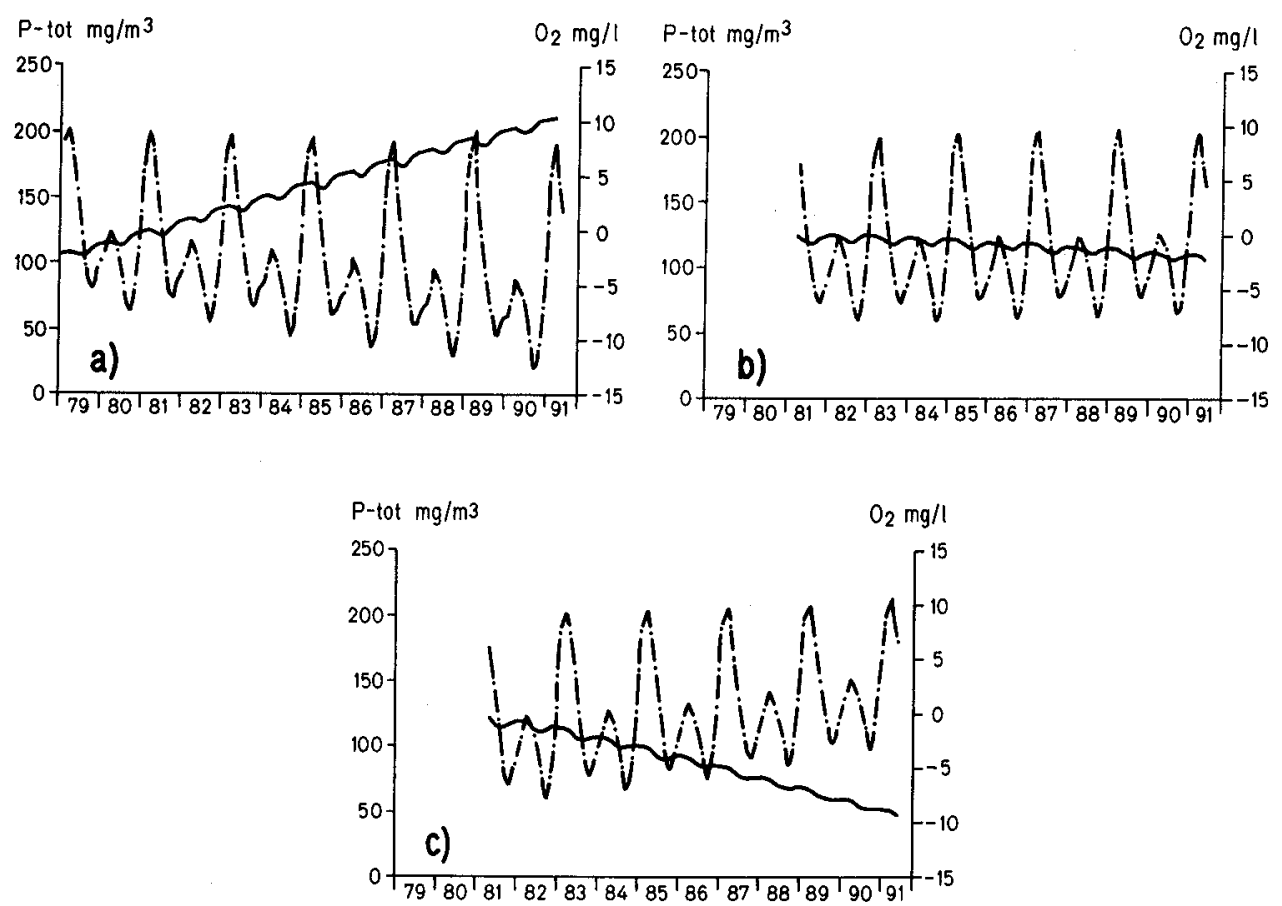

Abb.9. Modellrechnung: Veränderung der mittleren Gesamtphosphorkonzentration (-) und Sauerstoffkonzentration am Seegrund (---). a: Bei unveränderter Belastung. b: Bei Reduktion der Belastung um 50\%. c: Bei Reduktion der Belastung um 75\%.

Figure 9. Model calculation: Change in lake-average total phosphorus concentration (-) and oxygenconcentration (---) at a depth of $87 \mathrm{~m}$. a: Phosphorus loading $14.7 \mathrm{t} / \mathrm{yr}^{-1}$. b: Phosphorus loading $7.3 \mathrm{t} / \mathrm{yr}^{-1}$. $\mathrm{c}$ : Phosphorus loading $3.7 \mathrm{t} / \mathrm{yr}^{-1}$.

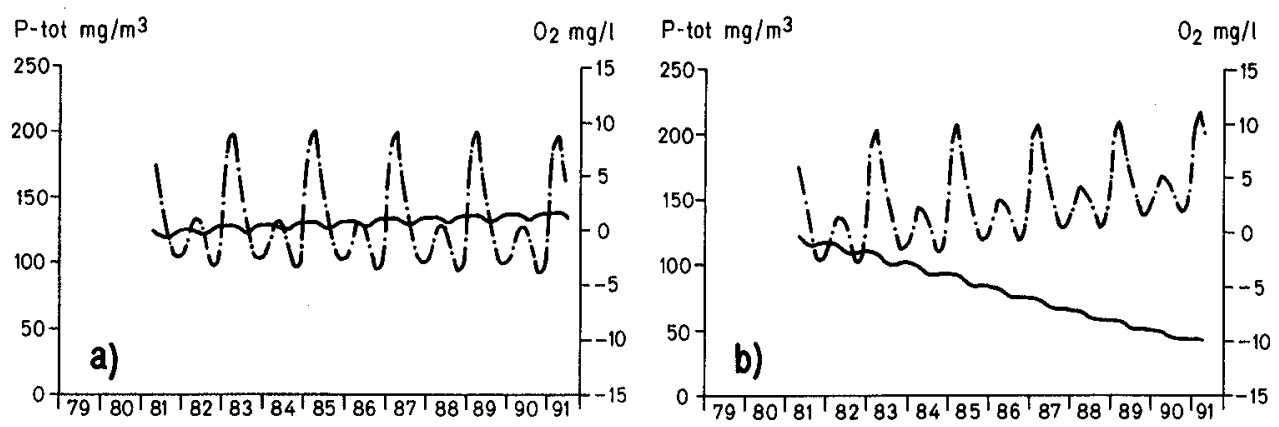

Abb. 10 Veränderung der mittleren Gesamtphosphorkonzentration $(-)$ und der Sauerstoffkonzentration am Seegrund (-- ). a: Bei $1,1 \mathrm{~m}^{3} / \mathrm{s}^{-1}$ Hypolimniondränage und unveränderter Phosphorbelastung. $b$ :

Bei $1,1 \mathrm{~m}^{3} / \mathrm{s}^{-1}$ Hypolimniondränage und um $50 \%$ reduzierter Phosphorbelastung.

Figure 10. Changes in lake-average total phosphorus concentration (-) and oxygen concentration (---) at a depth of $87 \mathrm{~m}$. a: Phosphorus loading $14.7 \mathrm{t} / \mathrm{yr}^{-1}$ and siphoning of $1.1 \mathrm{~m}^{3} / \mathrm{sec}^{-1}$ from a depth of

$87 \mathrm{~m}$. b: Phosphorus loading $7.3 \mathrm{t} / \mathrm{yr}^{-1}$ and siphoning of $1.1 \mathrm{~m}^{3} / \mathrm{sec}^{-1}$ from a depth of $87 \mathrm{~m}$. 
während der Sommerstagnation ein Absinken der Sauerstoffkonzentration unter $4 \mathrm{mg} \mathrm{O}_{2} / 1 \mathrm{zu}$ verhindern.

Seit 1954 sinken die Sauerstoffkonzentrationen am Seegrund während der zweiten Jahreshälfte regelmässig unter $4 \mathrm{mg} \mathrm{O}_{2} / 1$. Da dies die Phosphatfreisetzung an der Sediment-Wasser-Kontaktzone begünstigt (Abb.6), könnte durch Anheben der hypolimnischen Sauerstoffkonzentration die seeinterne Belastung reduziert werden. Wie Abb.11a zeigt, würde jedoch bei gleichbleibender externer Belastung die mittlere Phosporkonzentration im See nur unwesentlich niedriger. Für 1990/91 wird eine mittlere Phosphorkonzentration von rund $100 \mathrm{mg} / \mathrm{m}^{3}$ und eine Primärproduktion von $200 \mathrm{~g} \mathrm{C} \mathrm{m}^{2} \mathrm{Jahr}$ errechnet. Bei gleichzeitiger Reduktion der Phosphorbelastung um 50\% würden ähnlich gute Resultate erzielt wie mit der Hypolimniondränage (Abb. $11 \mathrm{~b}$ ).
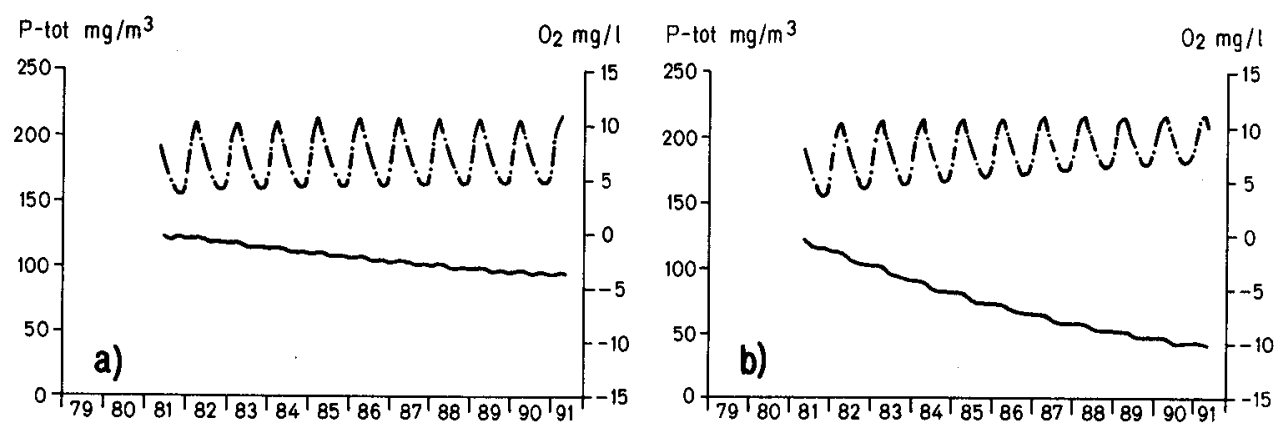

Abb. 11. Veränderung der mittleren Gesamtphosphorkonzentration (-) und der Sauerstoffkonzentration am Seegrund (---) beim Einsatz von vier Belüftungseinheiten (Bernhardt [2]). a: Bei unveränderter Phosphorbelastung. b: Bei um 50\% reduzierter Phosphorbelastung.

Figure 11. Change in lake-average total phosphorus concentration (-) and oxygen concentration (---) at a depth of $87 \mathrm{~m}$. a: Phosphorus loading $14.7 \mathrm{t} / \mathrm{yr}^{-1}$ and areation of the hypolimnion with four areation units according to Bernhard [2]. b: Phosphorus loading $7.3 \mathrm{t} / \mathrm{yr}^{-1}$ and areation of the hypolimnion with four areation units according to Bernhard [2].

Da ein Grossteil der Phosphorquellen diffus ist (s. S. 249), wäre es unrealistisch anzunehmen, dass die Phosphorbelastung des Sees kurzfristig um deutlich mehr als $50 \%$ reduziert werden könnte. Eine vollständige Sanierung des Sees durch eine Reduktion der Phosphorbelastung allein ist daher nicht möglich. Wie die Modellrechnungen zeigen, würden umgekehrt auch seeinterne Massnahmen (wie Hypolimnionbelüftung oder Hypolimniondränage) nicht ausreichen, das Sanierungsziel zu erreichen, wenn nicht gleichzeitig die Phosphorbelastung um wenigstens 50\% reduziert wird.

\section{Diskussion}

Wie Abb. 5 zeigt, steigt der totale P-Export (Sedimentation plus Export durch den Abfluss) proportional zur mittleren P-Konzentration im See, solange diese den Wert von ungefähr $30 \mathrm{mg} \mathrm{P} / \mathrm{m}^{3}$ nicht überschreitet. Bei dieser Konzentration erreicht der totale P-Export etwa $7 \mathrm{t} / \mathrm{Jahr}$. Somit könnte im stationären Zustand, wo jährlicher 
P-Input und P-Export einander gleich sind, der See gerade noch eine P-Belastung von $7 \mathrm{t} / \mathrm{Jahr}$ verkraften, ohne in jenen Konzentrationsbereich zu kommen, wo sich der totale P-Export auf einer Höhe von rund 7,5 t/Jahr stabilisiert. Bei grösseren PBelastungen steigt die P-Konzentration im See so lange an, bis auch der totale PExport wieder zunimmt und den Wert der Belastung erreicht. Diese Situation ist offenbar bei einer mittleren P-Konzentration von $100 \mathrm{mg} \mathrm{P} / \mathrm{m}^{3}$ noch nicht erreicht. Dies bedeutet, dass knapp oberhalb einer kritischen Belastung von rund $7 \mathrm{t} / \mathrm{Jahr}$ die Steady-state-P-Konzentration von knapp $30 \mathrm{mg} / \mathrm{m}^{3}$ sprunghaft auf mehr als $100 \mathrm{mg} / \mathrm{m}^{3}$ ansteigt.

In der Veränderung der P-Konzentration des Sees (Abb. 1) wird dieser Konzentrationssprung deshalb nicht manifest, weil es' bei einem Zufuhrüberschuss von wenigen Tonnen pro Jahr viele Jahre dauert, bis der Seeinhalt von knapp $20 \mathrm{t}$ auf mehr als $70 \mathrm{t}$ angewachsen ist. Da aber Export und Nettosedimentation das Plateau von 7,5 t im Mittel nicht überschreiten, ist der See bei der heutigen Phosphorbelastung von $14,7 \mathrm{t} / \mathrm{Jahr}$ offensichtlich nicht im Stationärzustand. Dies erklärt die Prognose des dynamischen Seemodells (Abb.9a), wonach die Phosphorkonzentration im See bei unveränderter Belastung noch weiter ansteigen wird.

Nach Abb. 5 kann unter günstigen Bedingungen die Nettosedimentationsrate den angenommenen Mittelwert weit übertreffen. Hohe Nettosedimentationsraten wären zu erwarten bei überdurchschnittlich hohen hypolimnischen Sauerstoffkonzentrationen oder beim Auftreten von Algengesellschaften, die besonders viel Phosphor speichern und rasch sedimentieren. Leider ist das vorhandene Datenmaterial nicht vollständig genug, um diese Vermutungen zu erhärten. Nimmt man jedoch an, die Ursache der hohen Nettosedimentationsraten der Jahre 1974 und 1975 liege in einer relativ guten Sauerstoffversorgung des Hypolimnions, so lässt sich die Summe von Nettosedimentation und Export nach Inbetriebnahme einer Hypolimnionbelüftung ungefähr abschätzen. Aus Abb. 12 folgt, in weitgehender Übereinstimmung mit den Prognosen des dynamischen Modells (vgl. Abb. 11), dass bei einer wirkungsvollen Belüftung und einer Zufuhr von 14,7 t/Jahr bzw. 7,3 t/Jahr die mittleren Phosphorkonzentrationen des Sees Werten von $70 \mathrm{mg} / \mathrm{m}^{3}$ bzw. $30 \mathrm{mg} / \mathrm{m}^{3}$ zustreben würden. In den Jahren 1967/68, 1968/69 und 1975/76 wurde die Gesamtphosphorkonzentration in verschiedenen Tiefen in monatlichen Abständen bestimmt. Ein Vergleich der mittleren Gesamtphosphorkonzentration am Seegrund mit jener an der Seeoberfläche ergibt, dass mit einer Hypolimniondränage der jährliche Phosphorexport verdoppelt bis verdreifacht werden könnte.

Damit kann die Summe von P-Nettosedimentation plus P-Export über die Hypolimniondränage in Abhängigkeit vom Phosphorinhalt des Sees geschätzt werden (Abb. 12). In guter Übereinstimmung mit den Prognosen des dynamischen Modells (Abb. 10) folgt, dass die Phosphorkonzentration im See bei gleichbleibender Belastung $(14,7 \mathrm{t} / \mathrm{J})$ trotz vollständiger Ableitung des Abflusses über eine Hypolimniondränage noch weiter ansteigen würde, bei gleichzeitiger Reduktion der Belastung auf 7,3 t/Jahr das Sanierungsziel aber erreicht werden könnte.

Diese einfachen Massenbilanzüberlegungen bestätigen die Resultate des dynamischen Seemodells und erhärten damit dessen Glaubwürdigkeit. Sie zeigen ferner, dass der See seit Jahren

a) nicht im stationären Zustand mit seiner Phosphorbelastung ist und 


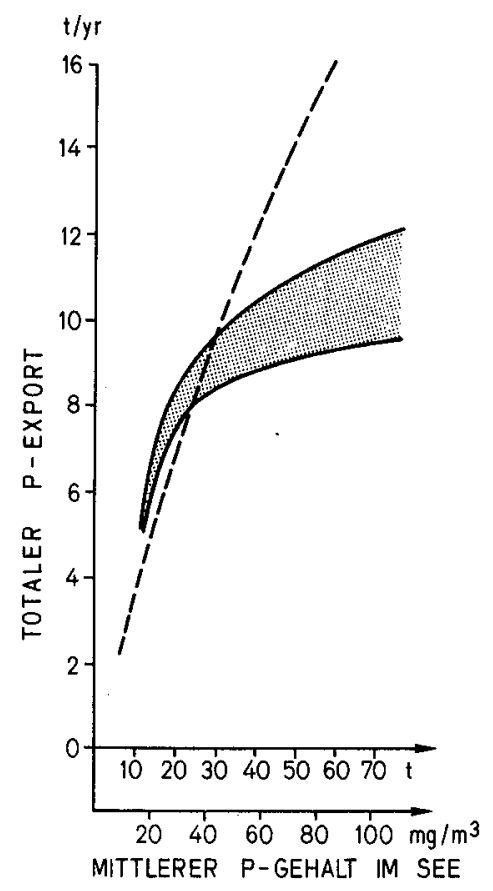

Abb. 12. Zusammenhang zwischen der Summe von Nettosedimentation plus Export via Abfluss (y-Axe) und mittlerem Phosphorgehalt des Sees (x-Axe). a: Bei Hypolimnionbelüftung (--).

Nettosedimentationsraten im aeroben Hypolimnion, hergeleitet aus Abb. 5. Weitere Erklärungen im Text. b: Bei Hypolimniondränage (gerasterte Fläche). Angenommene Exportraten bei Hypolimniondränage $=(2-3) \times a \times \Upsilon$. Weitere Erklärungen im Text.

Figure 12. Relationship between the sum of net deposition of phosphorus in the sediments plus export via the effluent (y-axis) and the average phosphorus content of the lake (x-axis). a: With areation of the hypolimnion (--) (maximum net deposition rates of phosphorus in an aerobic hypolimnion deduced from fig. 5). b: With siphoning of $1.1 \mathrm{~m}^{3} / \mathrm{s}$ from a depth of $87 \mathrm{~m}$ (shaded area). Loss of phosphorus via the siphon is assumed to be 2 to 3 times the natural phosphorus export.

b) zwischen dem Phosphorinhalt des Sees und der Phosphor-Nettosedimentationsrate kein linearer Zusammenhang besteht.

Die unkritische Anwendung von einfachen linearen, stationären Einboxmodellen müsste daher für diesen See zu falschen Schlüssen führen. In Abb. 13 wird die im Laufe der vergangenen 22 Jahre gemessene mittlere P-Konzentration im See (übernommen aus Abb.4) mit der zur jeweiligen Belastung gehörenden stationären Konzentration $[\mathrm{P}]_{\mathrm{st}}$ verglichen. Letztere wurde auf zwei Arten berechnet:

1. Das lineare stationäre Einboxmodell von Vollenweider [13] liefert den Ausdruck

$$
[\mathrm{P}]_{\mathrm{st}}=\frac{\mathrm{Z}}{\mathrm{Q}} \frac{\rho}{\rho+\sqrt{\mathrm{k} \rho}} \quad\left(\text { mit } \mathrm{k}=1 \mathrm{Jahr}^{-1}\right) .
$$

$\mathrm{Q}$ ist die Durchflussmenge $\left(3,8 \cdot 10^{7} \mathrm{~m}^{3} / \mathrm{Jahr}\right), \mathrm{Z}$ der jährliche P-Input in den See $(\mathrm{t} / \mathrm{Jahr})$ und $\rho$ die Durchflussrate, d.h. der jährliche relative Wasseraustausch 


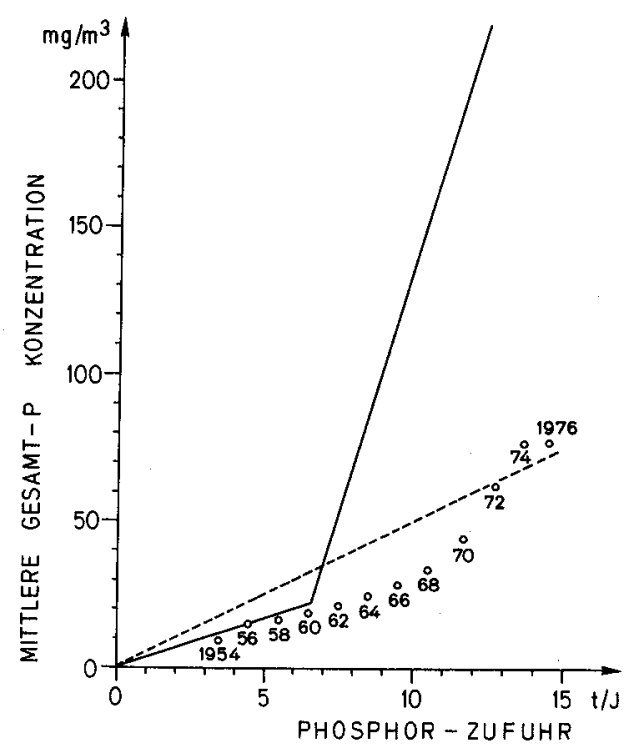

Abb. 13. Zusammenhang zwischen Gesamtphosphorkonzentration und Phosphorbelastung. Gemessene mittlere Gesamtphosphorkonzentrationen (o). Mit Hilfe von Massenbilanzüberlegungen $(\mathrm{Gl} .8,9)$ hergeleitete stationäre Konzentration (---). Mit Hilfe eines linearen Einboxmodells (Gl.4) hergeleitete stationäre Konzentrationen (...).

Figure 13. Observed averate concentrations of total phosphorus (o). Steady state phosphorus concentrations estimated from mass balance considerations (s. eq. 8, 9) (---). Steady state phosphorus concentrations estimated with a linear one-box model (eq. 4) (...). Relationship between phosphorus concentration and phosphorus loading.

(0,057/Jahr). Der Ausdruck $\sqrt{\mathrm{k} \rho}$ ist Vollenweiders Ansatz für die Nettosedimentationsrate, wobei der Autor aufgrund einer statistischen Analyse verschiedener Seedaten die Konstante $\mathrm{k} \mathrm{zu}_{1} \mathrm{Jahr}^{-1}$ bestimmt. Vollenweiders Modell ergibt einen linearen Zusammenhang zwischen $[\mathrm{P}]_{s t}$ und $\mathrm{Z}$; die entsprechende Gerade ist in Abb. 13 punktiert eingezeichnet.

2. Nimmt man an, der lineare Zusammenhang zwischen P-Menge im See und P-Export im Abfluss (Gl.2) gelte für alle P-Konzentrationen, so lässt sich die stationäre P-Bilanz schreiben:

$$
\mathrm{Z}=\mathrm{S}+\alpha \mathrm{I}_{\mathrm{st}}=\mathrm{S}+\frac{\alpha}{\rho}[\mathrm{P}]_{\mathrm{st}} \mathrm{Q},
$$

wobei die Beziehungen $I_{\mathrm{st}}=\mathrm{V}[\mathrm{P}]_{\mathrm{st}}$ und $\rho=\mathrm{Q} / \mathrm{V}$ benutzt worden sind. Auflösen von Gl. (5) nach $[\mathrm{P}]_{\mathrm{st}}$ ergibt

$$
[\mathrm{P}]_{\mathrm{st}}=\frac{\mathrm{Z}-\mathrm{S}}{\frac{a}{\rho} \mathrm{Q}} \text {. }
$$

Die Nettosedimentationsrate $S$ hängt ihrerseits von $[\mathrm{P}]_{\mathrm{st}} \mathrm{ab}$. 
Basierend auf Abb. 5 nehmen wir an, S steige bis zu einer mittleren P-Konzentration von $24 \mathrm{mg} / \mathrm{m}^{3}$ linear und nehme für höhere Konzentrationen den konstanten Wert von $6 \mathrm{t} / \mathrm{Jahr}$ an.

Für $[\mathrm{P}] \leq 24 \mathrm{mg} / \mathrm{m}^{3}$ gilt somit

$$
\mathrm{S}=\sigma[\mathrm{P}] .
$$

In (5) eingesetzt und nach $[\mathrm{P}]_{\mathrm{st}}$ aufgelöst ergibt

$$
[\mathrm{P}]_{\mathrm{st}}=\frac{\mathrm{Z}}{\sigma+\frac{a}{\rho} \mathrm{Q}} .
$$

Für $\sigma$ lässt sich aus Abb. 5 ein Wert von $0,25 \cdot 10^{9} \mathrm{~m}^{3} / \mathrm{Jahr}$ ermitteln. Für $[\mathrm{P}] \geq 24 \mathrm{mg} / \mathrm{m}^{3}$ ergibt sich aus (6):

$$
[\mathrm{P}]_{\mathrm{st}}=\frac{\mathrm{Z}}{\frac{a}{\rho} \mathrm{Q}}-\frac{6 \mathrm{t} / \mathrm{Jahr}}{\frac{\alpha}{\rho} \mathrm{Q}} .
$$

Der nach diesem Ansatz (Gl. 8 und 9) berechnete Zusammenhang zwischen $[\mathrm{P}]_{\text {st }}$ und $\mathrm{Z}$ ist in Abb. 13 als ausgezogene Linie dargestellt. Gleichzeitig zeigt Abb. 13, wie die Phosphorkonzentration im See als Folge der stetig anwachsenden Belastung zugenommen hat.

Bis zum Jahre 1960 war die P-Konzentration im See praktisch im Gleichgewicht mit der damaligen Phosphorbelastung. Seither sind die beobachteten P-Konzentrationen deutlich hinter den zur jeweiligen Belastung gehörenden stationären Werten zurückgeblieben.

Die nach dem Ansatz von Vollenweider [13] berechneten stationären Werte liegen für kleine Belastungen über, für grössere Belastungen aber erheblich unter den mittels des nichtlinearen Modells (5) berechneten Werten. Dieses Beispiel macht deutlich, dass einfache Modelle, welche das Verhalten eines «mittleren» Sees beschreiben, nicht unkritisch auf jeden konkreten Fall übertragen werden können.

\section{Empfehlungen}

Um eine weitere Verschlechterung des Seezustandes zu verhindern, empfehlen wir aufgrund der oben angeführten Resultate und der positiven Erfahrungen, die während des Winters 1982 im Baldeggersee mit der Zwangszirkulation des Sees gemacht wurden, die folgenden Massnahmen möglichst rasch zu realisieren:

- Den See während des Winters durch Einblasen von Luft in eine Zwangszirkulation zu versetzen, mit dem Ziel, die Sauerstoffkonzentration über dem Seegrund bis zum Eintreten der Stagnationsphase alljährlich auf mindestens $10 \mathrm{mg} / 1$ anzuheben. 
- Im See möglichst bald eine Hypolimniondränageleitung zu installieren oder durch Belüftung oder Begasung mit reinem Sauerstoff dafür zu sorgen, dass die Sauerstoffkonzentration nicht mehr unter $4 \mathrm{mg} \mathrm{O}_{2} / 1$ absinkt.

- Im Einzugsgebiet des Sees durch ein sorgfältiges Abschreiten sämtlicher Zuflüsse punktförmige Phosphorbelastungsquellen zu eruieren und möglichst rasch zu eliminieren.

- Durch intensive Aufklärung die Bauern zum sorgfältigen Umgang mit dem Hofdünger zu motivieren (z.B. keine Düngung auf Brache, auf durchnässten oder gefrorenen Boden).

- Durch betriebliche Erhebungen abzuklären, ob die vorhandene Jauchegrubenkapazität und die zur Verfügung stehende Austragsfläche in Übereinstimmung stehen mit dem vorhandenen Tierbestand.

- Den Tierbestand an die gegebenen Kapazitäten anzupassen.

Diese Arbeit zeigt, dass es auch in grossen Seen möglich ist, durch seeinterne Massnahmen die Wasserqualität günstig zu beeinflussen. Es muss aber unterstrichen werden, dass derartige Massnahmen nur so lange helfen, als die Anlagen auch in Betrieb sind. Da es im Falle des Sempachersees selbst mit grössten Anstrengungen nicht möglich sein wird, die jährliche Phosphorbelastung kurzfristig unter den kritischen Wert von 7,5 t zu reduzieren, wird ein dauernd anhaltender Einsatz der gewählten seeinternen Massnahmen unumgänglich sein. Aus diesem Grund wäre wahrscheinlich der Variante «Hypolimniondränage» der Vorzug zu geben, da ihr Betrieb weniger Energie und wahrscheinlich auch weniger Unterhaltsarbeiten erfordert als eine Hypolimnionbelüftung.

\section{Zusammenfassung}

Seit 1954 hat sich die mittlere Phosphatkonzentration im Sempachersee verzwanzigfacht (s. Abb. 1) und die Gesamtphosphorkonzentration verachtfacht. Es wird gezeigt, dass der See nicht im Steady state mit seiner externen Belastung ist, und dass die Nettosedimentation des Phosphors nicht linear mit dem Phosphorinhalt des Sees anwächst (s. Abb.5). Lineare Steady-state-Einboxmodelle können daher nicht auf diesem See angewandt werden. Mit Hilfe eines nichtlinearen dynamischen Seemodells $(5,9)$ wird prognostiziert, dass die definierten Gewässerschutzziele ([P] $\left.\leqslant 30 \mathrm{mg} \mathrm{m}^{-3},\left[\mathrm{O}_{2}\right] \geqslant 4 \mathrm{mg} \mathrm{O}_{2} / 1\right)$ innerhalb der nächsten 15 Jahre nur erreicht werden können, wenn die externe Phosphorbelastung um mindenstens $50 \%$ reduziert wird und gleichzeitig seeinterne Massnahmen ergriffen werden (Hypolimniondränage oder Belüftung des Hypolimnions).

\section{SUMMARY}

Lake Sempach is situated in the central part of Switzerland some $15 \mathrm{~km}$ northwest of Lucerne. Its surface area, maximum and mean depth are $14.4 \mathrm{~km}^{2}, 87 \mathrm{~m}$ and $47 \mathrm{~m}$ respectively..Its average discharge yields $1.2 \mathrm{~m}^{3} \mathrm{~s}^{-1}$. About $52 \mathrm{~km}^{2}$ of the drainage area are used for agricultural purposes, $10 \mathrm{~km}^{2}$ are forested. About 10,000 people inhabit the drainage basin of the lake.

Since 1954 average phosphate and total phosphorus concentration have increased twenty- and eightfold respectively (see figs. 1 and 4). As a consequence of increased primary production $\mathrm{pH}$ values, supersatu- 
ration of oxygen and differences in alkalinity between winter and summer increased in the epilimnion. Concomitantly, measurements taken in October, show increasing concentrations of ammonia and manganese as well as a decrease of oxygen concentrations in the deep hypolimnion (see fig. 2).

It is the goal of this work

- to identify and to quantify the various phosphorus sources,

- to establish the relationship between lake external phosphorus loading and phosphorus concentration in the lake,

- to predict reactions of the lake to a reduced phosphorus loading and (or) to lake internal restoration measures, such as areation or siphoning of the hypolimnic water.

The phosphorus loading of the lake was estimated three times since 1954. It was in the order of $3.4 \mathrm{t} / \mathrm{yr}$ in $1954,9.2 \mathrm{t} / \mathrm{yr}$ in $1966 / 67$ and $14.7 \mathrm{t} / \mathrm{yr}$ in 1976/77. This clearly shows that the construction of wastewater purification plants which started in 1968 was insufficient to reduce the total phosphorus loading, although in $197758 \%$ of the population was connected to wastewater treatment plants. Reasons are simultaneous growth of population density, increasing phosphorus content in the sewage due to polyphosphates in detergents and increasing phosphorus run-off and -eluation from land used for agricultural purposes.

In 1977 sewage contributed 5.5 to $9.3 \mathrm{t} / \mathrm{yr}$, agriculture 4.2 to $8.0 \mathrm{t} / \mathrm{yr}$ and precipitation $1.2 \mathrm{t} / \mathrm{yr}$. From this it is conlcuded that the total phosphorus loading cannot be lowered by more than $50 \%$ in the nearer future.

The phosphorus balance of a lake is described by the equation

$$
\frac{\mathrm{dI}}{\mathrm{dt}}=\mathrm{Z}-\mathrm{E}-\mathrm{S},
$$

where I means the phosphorus content of the lake, Z, E and S phosphorus input, export of phosphorus via the effluent and net deposition of phosphorus in the sediments of the lake respectively. Figure 3 shows that $\mathrm{E}$ is linearely related to $\mathrm{I}$. Hence the yearly net deposition rate of phosphorus can be expressed as

$$
\mathrm{S}=\mathrm{Z}-a \cdot \mathrm{I}-\frac{\mathrm{dI}}{\mathrm{dt}}
$$

where $\alpha=0.042 \mathrm{yr}^{-1}$. Using equation (2) and the information about $\mathrm{Z}$ and $\mathrm{dI} / \mathrm{dt}$ summarized in figure 4 yearly averages of $S$ were estimated for the period 1954 to 1979 and plotted versus $I$ in figure 5 . This plot clearly indicates that the relationship between net deposition of phosphorus and the phosphorus content of the lake is not simply a linear one, as it is assumed in well-known linear phosphorus models (e.g. Vollenweider [14]). Although above a critical level of $I=20 \mathrm{t}$ (corresponding to an average phosphorus concentration of about $30 \mathrm{mg} \mathrm{m}^{-3}$ ) points start to scatter widely, it becomes evident from figure 5 that above this level $\mathrm{S}$ does rather decrease than increase with increasing $\mathrm{I}$.

A lake is in cyclic steady state if the yearly phosphorus input equals the sum of net deposition plus export via the effluent. Since as shown in figure 5 on an average base this sum seems not to exceed $8 \mathrm{t} / \mathrm{yr}^{-1}$ the lake obviously is not in steady state with its phosphorus loading of $14.7 \mathrm{t} / \mathrm{yr}^{-1}$

Figure 13 compares steady state concentrations estimated from massbalances with those calculated using a linear phosphorus model suggested by Vollenweider [13]. Obviously since 1961 the linear model underestimates the true steady state and since 1972 it even underestimates actually observed non steady state concentrations, allthough the phosphorus loading increased continuously.

Hence linear steady state phosphorus models cannot be applied to lake Sempach, because

a) $\mathrm{S}$ and $\mathrm{I}$ are not linearly related.

b) The lake is not in steady state.

To estimate the effects of decreasing the phosphorus loading, hypolimnion areation or hypolimnion siphoning, a modified lake model of Imboden and Gächter [9] shown in figure 7 was applied. Figures 9 , 10 and 11 show that the goals of lake water quality (average phosphorus concentration less than $30 \mathrm{mg} \mathrm{m}^{-3}$ and oxygen concentration at no depth and at no time below $4 \mathrm{mg} \mathrm{l}^{-1}$ ) cannot be achieved, neither with a reduction of the phosphorus loading by $50 \%$ nor by hypolimnic areation nor by siphoning of the total discharge $\left(1.1 \mathrm{~m}^{3} \mathrm{~s}^{-1}\right)$ from the deepest point of the lake. To reach these goals a combination of a $50 \%$ reduction of the loading with one of the two lake internal measures is necessary. Siphoning is 
recommended in preference to areation since this lake internal measure requires much less energy and maintenance.

\section{RÉSUMÉ}

Mesures possibles d'assainissement du lac de Sempach

Depuis 1954, la concentration moyenne de phosphate dans le lac de Sempach a augmenté de 20 fois (fig. 1) et la concentration totale de phosphore est actuellement 8 fois plus forte. Il a été démontré que le lac n'est pas en «steady state» avec sa charge externe et que la sédimentation nette du phosphore n'augmente pas linéairement avec la teneur en phosphore du lac (fig.5). De ce fait, des modèles à boîte simple de «steady state» linéaires ne peuvent pas être appliqués à ce lac. Avec l'aide d'un modèle dynamique non linéaire de lac $(5,9)$, on prévoit que les buts définis de la protection des eaux $[(P)$ $\leqslant 30 \mathrm{mgm}^{-3},\left(\mathrm{O}_{2}\right) \geqslant 4 \mathrm{mg} \mathrm{O}_{2} / 1$ ] ne pourront être atteints pendant les 15 prochaines années que si la charge externe de phosphore est réduite d'au moins $50 \%$ et que simultanément les mesures internes d'assainissement sont prises (drainage ou aération de l'hypolimnion).

\section{LITERATURVERZEICHNIS}

1 AGBA: Die Belastung von Boden und Gewässern durch die Landwirtschaft im Einzugsgebiet des Sempachersees unter besonderer Berücksichtigung des Phosphatgehalts. Gutachten der Arbeitsgemeinschaft Beratender Agronomen AGBA AG im Auftrag des Kantons Luzern 54 (1980).

2 Bernhardt, H.: Die hypolimnische Belüftung der Wahnbachtalsperre. GWF-Wasser/Abwasser, 119 (4), 177-182 (1978).

3 Casiraghi, R.: Diplomarbeit, Abendtechnikum der Innerschweiz (1978).

4 Du Bois, A.M., und Geigy R.: Beiträge zu Ökologie, Fortpflanzungsbiologie und Metamorphose von Sialis lutaria L. Rev. suisse Zool. 42, 169-248 (1935).

5 EAWAG: Gutachten über die Sanierungsmöglichkeiten des Sempachersees. Gutachten Nr. 4564, 67 Seiten (1979)

6 Gächter, R., und Furrer, O.: Der Beitrag der Landwirtschaft zur Eutrophierung der Gewässer in der Schweiz. Schweiz. Z. Hydrol. 34, 41-70 (1972).

7 Heuscher, J.: Der Sempachersee und seine Fischereiverhältnisse. Schweiz. FischZtg 3, 1-51 (1895).

8 Sly, P. G.: Lake Erie and its Basin. J. Fish. Res. Bd Canada 33, 355-370 (1976).

9 Imboden, D., und Gächter, R.: A dynamic lake model for trophic state prediction. J. Ecol. Modelling 4, 77-98 (1978).

10 Redfield, A.C., Ketchum, B.H., und Richards, F.A., in: Hill, M.N. (Ed.): The Sea, Vol. 2, S. 26. Wiley-Intersciences, New York 1966.

11 Stadelmann, P.: Der Zustand des Sempachersees. Wasser, Energie, Luft 72, 311-318 (1980).

12 Verordnung über Abwasserleitungen vom 8. Dez. 1975 (Stand am 1. April 1977).

13 Vollenweider, R.A.: Advances in defining critical loading levels for phosphorus in lake eutrophication. Memorie Ist. ital. Idrobiol. 33, 53-83 (1976).

14 Züllig, H.: Beobachtungen über die zunehmende Eutrophierung des Sempachersees. Plan, Schweiz. Z. Landes-, Regional- und Ortsplanung, Heft Nr. 3, 100-103 (1958).

Adresse der Autoren: Seenforschungslaboratorium EAWAG/ETH, CH-6047 Kastanienbaum, Schweiz. 